\title{
Evidence of the Gaia-VLBI position differences being related to radio source structure
}

\author{
Ming H. Xu1 ${ }^{1,2,4}$, Susanne Lunz ${ }^{3}$, James M. Anderson ${ }^{4}$, Tuomas Savolainen ${ }^{1,2}$, Nataliya Zubko ${ }^{5}$, and Harald Schuh ${ }^{4,3}$ \\ 1 Aalto University Metsähovi Radio Observatory, Metsähovintie 114, 02540 Kylmälä, Finland \\ e-mail: minghui .xu@aalto.fi \\ 2 Aalto University Department of Electronics and Nanoengineering, PL15500, 00076 Aalto, Finland \\ 3 DeutschesGeoForschungsZentrum (GFZ), Potsdam, Telegrafenberg, 14473 Potsdam, Germany \\ 4 Institute of Geodesy and Geoinformation Science, Technische Universität Berlin, Straße des 17. Juni 135, 10623 Berlin, Germany \\ 5 Finnish Geospatial Research Institute, Geodeetinrinne 2, 02430 Masala, Finland
}

Received 18 December 2020 / Accepted 27 January 2021

\begin{abstract}
Context. We report the relationship between the Gaia-VLBI position differences and the magnitudes of source structure effects in VLBI observations.

Aims. Because the Gaia-VLBI position differences are statistically significant for a considerable number of common sources, we discuss and attempt to explain these position differences based on VLBI observations and available source images at centimeter wavelengths.

Methods. Based on the derived closure amplitude root mean square (CARMS), which quantifies the magnitudes of source structure effects in the VLBI observations used for building the third realization of the International Celestial Reference Frame, the arc lengths and normalized arc lengths of the position differences are examined in detail. The radio-jet directions and the directions of the GaiaVLBI position differences are investigated for a small sample of sources.

Results. Both the arc lengths and normalized arc lengths of the Gaia and VLBI positions are found to increase with the CARMS values. The majority of the sources with statistically significant position differences are associated with the sources having extended structure. Radio source structure is the one of the major factors of these position differences, and it can be the dominant factor for a number of sources. The vectors of the Gaia and VLBI position differences are parallel to the radio-jet directions, which is confirmed via stronger evidence.
\end{abstract}

Key words. galaxies: active - galaxies: jets - astrometry - reference systems - radio continuum: galaxies

\section{Introduction}

The International Celestial Reference Frame (ICRF) was adopted as the Fundamental Celestial Reference Frame for astronomy in January 1998 by the International Astronomical Union (IAU) (Ma et al. 1998). The ICRF is realized by the positions of distant radio sources, mostly active galactic nuclei (AGNs), based on the astrometric and geodetic very long baseline interferometry (VLBI) observations coordinated by the International VLBI Service for Geodesy and Astrometry (IVS; Schuh \& Behrend 2012; Nothnagel et al. 2017, see also the IVS website ${ }^{1}$, and relies on the VLBI technique for its maintenance and improvement. As officially adopted by the IAU in January 2019, the third realization of the ICRF (ICRF3; Charlot et al. 2020) was established based on 40 years of VLBI observations and, for the first time, independently at three different radio frequencies. The radio source positions in the ICRF3 have accuracies at submilliarcsecond (sub-mas) levels. The European Space Agency mission Gaia $^{2}$ (Gaia Collaboration 2016) has released position estimates and other astrometric parameters for the celestial objects with optical $G$ magnitudes $<21$ mag based on the observations during the 22 months since July 2014 (DR2; Gaia Collaboration 2018a). The

1 https://ivscc.gsfc.nasa.gov/index.html

2 https://sci.esa.int/web/gaia color-dependent calibration is possible based on the Gaia DR2, and leads to improvements in the astrometric solution thereafter (Lindegren et al. 2021). The Gaia Early Data Release 3 (EDR3; Gaia Collaboration 2021) has made the data available based on the first 34 months of its observations.

A good overall agreement between radio and optical positions was achieved for the cross-matched common objects (Mignard et al. 2016; Gaia Collaboration 2018b); the median arc length between the source positions from Gaia and VLBI is $\sim 0.5$ mas based on the Gaia DR2 and the ICRF3. However, the distribution of the arc lengths between radio and optical positions normalized by their uncertainties (hereafter normalized arc length) deviates from the expected Rayleigh distribution with $\sigma=1$. The most obvious deviations in that distribution are the long tail spreading to very large normalized arc lengths and the significant deficit of values in the bins around the expected peak. In the Gaia DR1, there were only a small percentage of sources with normalized arc lengths >3 (Mignard et al. 2016; Petrov \& Kovalev 2017a). In the Gaia DR2, the fraction of these sources increases to more than 10\% (Petrov \& Kovalev 2017b; Gaia Collaboration 2018b; Petrov et al. 2019; Makarov et al. 2019). By deselecting objects mostly based on the optical properties, Makarov et al. (2019) still found that $20 \%$ of the sources had normalized arc lengths $>3$. The factors causing these position differences between 
optical and radio are still uncertain, even though there are a variety of possible astrophysical explanations (Makarov et al. 2019; Plavin et al. 2019a; Kovalev et al. 2020). For instance, Kovalev et al. (2017) and Petrov et al. (2019) suggested that the main cause of the position differences is optical structure, the optical jets at milliarcsecond scales. Understanding these position differences is very important because (1) it will lead to a better selection of the common sources for aligning the optical frame to the radio frame; (2) the number of sources with statistically significant position differences can continue to increase in future Gaia data releases, which would allow more small position differences to be detected at the $3 \sigma$ confidence level; and (3) the position differences may tell us something important about the astrophysics of AGNs.

We examine the position differences between Gaia and VLBI from the radio side. As demonstrated in the imaging survey of radio sources (Charlot 1990a; Fey \& Charlot 1997), the celestial reference frame (CRF) sources commonly have angular structure on milliarcsecond scales at centimeter wavelengths ${ }^{3}$. Source structure is time and frequency dependent, and it is not modeled in the data analysis of building the ICRF3. The position estimates and their uncertainties in the ICRF3 are based on global least-squares fitting (LSQ), and are thus not able to characterize the impacts of the systematical position variations over the 40 years due to source structure. For example, the position uncertainties from LSQ are likely underestimated in the presence of systematic errors. Our previous study used the same VLBI observations as for the creation of the ICRF3 to quantify the magnitude of effects of source structure on VLBI observables for each individual source (Xu et al. 2019). In this paper we apply the results to investigate the relationship between the Gaia-VLBI position differences and source structure at centimeter wavelengths. We then attempt to explain and discuss these position differences based on the radio images from the Monitoring Of Jets in Active galactic nuclei with VLBA Experiments (MOJAVE; Lister et al. 2018).

The paper is structured as follows. We introduce in Sect. 2 how the arc lengths of position differences, the normalized arc lengths, and the quantitative values of measuring structure effects are derived. We describe in Sect. 3 the results from the examination of arc lengths, normalized arc lengths, optical $G$ magnitudes, and redshifts with respect to source structure. In Sect. 4.1 we discuss the effects of source structure and its quantification, in Sect. 4.2 the frequency-dependent shift of the VLBI core position, in Sect. 4.3 the large position differences that are statistically significant, in Sect. 4.4 the magnitudes of the position differences, and in Sect. 4.5 the directions of the position differences. We present our conclusion in Sect. 5.

\section{Data}

\subsection{Source positions from Gaia and VLBI}

We used the right ascension and declination estimates, their uncertainties, and the correlations between these two coordinates in the ICRF3 ${ }^{4}$, which contains 4536 sources observed by astrometric and geodetic VLBI at $S / X$ band. The median uncertainties of right ascension and declination reported in the ICRF3 are 0.155 mas and 0.217 mas, respectively. We used the Gaia DR2

\footnotetext{
3 Images of CRF sources can be found at http://www.physics. purdue.edu/astro/MOJAVE/

4 http://hpiers.obspm.fr/icrs-pc/newwww/icrf/icrf3sx. txt
}

and EDR $3^{5}$ to obtain the five astrometric parameters (source position, proper motion, and parallax), their uncertainties, the correlations between them, and the optical magnitude.

Even though the cross-match between radio and optical catalogs basically relies on the position coincidence, other criteria are needed to reduce the risk of false matches. Lindegren et al. (2018) applied constraints on the other three astrometric parameters and the number of observations, and masked out the region near the Galactic plane, as shown in Eq. (13) of the publication. Petrov \& Kovalev (2017a) used the concept of probability of false association as a function of Gaia source density on a regular grid and the possible area defined by the positions and the uncertainties at radio and optical wavelengths for each potential match. We combined these two methods to identify the common objects between the ICRF3 and the Gaia DR2, which gives 2970 sources (Lunz et al. 2019, please refer to the poster ${ }^{6}$ ). Based on the Gaia EDR3 and the ICRF3, we identified 3142 common sources, the same number of matched sources as found by the Gaia team in the ongoing analysis (François Mignard, priv. comm.).

For each common source we calculated the arc length between the Gaia and VLBI positions $\rho$ by

$\rho=\sqrt{\left(\Delta_{\alpha} \cos \delta\right)^{2}+\Delta_{\delta}^{2}}$

where $\Delta_{\alpha}$ and $\Delta_{\delta}$ are the differences of right ascension and declination in the Gaia data and the ICRF3, respectively, and $\delta$ is the declination. The normalized arc length $X_{\rho}$ is defined and calculated by

$X_{\rho}=\rho / \sigma_{\rho}$,

where $\sigma_{\rho}$ is the uncertainty of $\rho$ based on the full $2 \times 2$ covariance matrix, as described in Eqs. (4) and (5) of Mignard et al. (2016).

To characterize the position uncertainty with a single value, the semi-major axis of the error ellipse $\sigma_{\text {pos, max }}$ was computed for both Gaia and VLBI by

$$
\begin{aligned}
\sigma_{\text {pos }, \max }^{2}= & \frac{1}{2}\left[\left(\sigma_{\alpha} \cos \delta\right)^{2}+\sigma_{\delta}^{2}\right. \\
& \left.+\sqrt{\left(\left(\sigma_{\alpha} \cos \delta\right)^{2}-\sigma_{\delta}^{2}\right)^{2}+\left(2 \mathrm{C}_{\alpha \delta} \sigma_{\alpha} \cos \delta \sigma_{\delta}\right)^{2}}\right]
\end{aligned}
$$

where $\sigma_{\alpha}$ and $\sigma_{\delta}$ are the uncertainties of right ascension and declination, respectively, and $\mathrm{C}_{\alpha \delta}$ is the correlation coefficient of the two coordinates.

Based on the Gaia DR2 and the ICRF3, there are 732 sources with $X_{\rho}>3.0$; based on the Gaia EDR3, 804 sources have $X_{\rho}>$ 3.0.

\subsection{Closure amplitude root mean square (CARMS)}

We adopted the log closure amplitude root mean square (CARMS) values from Table 2 in Xu et al. (2019) to quantify the magnitude of source structure effects for each individual source ${ }^{7}$.

Due to the missing data for calibration and the insensitivity of the parameters of geodetic concern, visibility amplitudes from interferometry were not used for most of the geodetic

\footnotetext{
5 https://gea.esac.esa.int/archive/

6 http://wWw.oan.es/evga2019/EVGA2019_PDF/P310_ EVGA2019_Lunz.pdf

7 The complete table is available through the CANFAR data DOI at: https: //WWW . canfar . net/citation/landing?doi=20.0010
} 
Table 1. Arc lengths $\rho$ and normalized arc lengths $X_{\rho}$ with respect to CARMS.

\begin{tabular}{|c|c|c|c|c|c|c|c|}
\hline \multirow[t]{2}{*}{ CARMS } & \multirow[t]{2}{*}{$N_{\text {src }}$} & \multicolumn{2}{|c|}{$\rho$ [mas] } & \multicolumn{2}{|c|}{$X_{\rho}$} & \multicolumn{2}{|c|}{$\sigma_{\rho}[\mathrm{mas}]$} \\
\hline & & Mean & Median & Mean & Median & Mean & Median \\
\hline$<0.10$ & 207 & 0.717 & 0.459 & 1.825 & 1.492 & 0.382 & 0.304 \\
\hline$[0.10-0.20)$ & 724 & 0.710 & 0.448 & 2.135 & 1.600 & 0.350 & 0.269 \\
\hline$[0.20-0.30)$ & 617 & 0.772 & 0.418 & 2.845 & 1.751 & 0.297 & 0.229 \\
\hline$[0.30-0.40)$ & 334 & 1.080 & 0.411 & 3.665 & 1.876 & 0.287 & 0.218 \\
\hline$[0.40-0.50)$ & 220 & 1.347 & 0.452 & 5.425 & 2.543 & 0.258 & 0.189 \\
\hline$[0.50-0.60)$ & 128 & 1.238 & 0.483 & 5.286 & 2.396 & 0.268 & 0.215 \\
\hline$[0.60-0.70)$ & 87 & 1.634 & 0.845 & 6.273 & 3.747 & 0.324 & 0.252 \\
\hline$[0.70-0.80)$ & 59 & 1.536 & 0.769 & 7.918 & 3.842 & 0.268 & 0.173 \\
\hline$[0.80-0.90)$ & 35 & 3.516 & 1.081 & 12.247 & 5.686 & 0.327 & 0.245 \\
\hline$\geq 0.90$ & 49 & 3.660 & 1.334 & 14.502 & 6.894 & 0.276 & 0.204 \\
\hline All & 2460 & 1.012 & 0.459 & 3.628 & 1.843 & 0.314 & 0.240 \\
\hline
\end{tabular}

VLBI observations. However, they carry valuable information about source angular structure, which causes structure effects in group delays up to hundreds of picoseconds (Charlot 1990b; $\mathrm{Xu}$ et al. 2016). By forming quadrangles with four baselines, we obtain a ratio of the four amplitude observables to cancel out exactly the station-based errors, which is called closure amplitude and provides information about the intrinsic source structure. For an ideal point-like source, all the baselines will observe the same amplitude within the thermal noise, giving closure amplitudes close to unity; for an extended source the closure amplitudes deviate from unity. The CARMS value of a source is defined to be the root mean square (rms) of $\log$ closure amplitudes at the $X$ band, based on the basic weighting scheme (see Eqs. (2)-(4) and (6)-(8) in Xu et al. 2019). In addition to the study in Xu et al. (2019), supporting material is also available ${ }^{8}$, where the closure phase and closure amplitude plots are available for tens of sources to demonstrate the source structure effects and compare with their CARMS values.

The CARMS values are available for 3417 radio sources in the ICRF3, and were derived from the astrometric and geodetic VLBI observations from 1979 to 2018, the same dataset establishing the ICRF3. They are in the range 0.03-1.63, and the mean and median values are 0.31 and 0.24 , respectively. The CARMS values generally classify the CRF sources into three categories:

1. CARMS $<0.2$ indicates minimum structure;

2. CARMS $>0.3$ indicates significant structure;

3. CARMS $>0.4$ indicates very extended structure.

The CARMS values were validated by the different source categories in the ICRF catalogs. For instance, the 39 special handling sources in the second realization of the ICRF (Fey et al. 2015), which have variations in the time series of VLBI position estimates at mas or sub-mas levels, have the median CARMS of 0.60 , while the median value for the ICRF3 defining sources, used to define the axis directions of the ICRF3, is 0.25. Recently, these CARMS values were used to select radio sources with minimum structure to assess the quality of group delays in the broadband VLBI system (Xu et al. 2021a).

For the 3142 common sources from the Gaia EDR3, the CARMS values are available for 2460 sources, or $78 \%$; the mean and median CARMS values are 0.30 and 0.24 , respectively, which are at the same level as those of the 3417 sources. We examined the source position estimates based on both the Gaia

\footnotetext{
8 https://www. canfar.net/citation/landing?doi=19.0007
}

DR2 and EDR3, but we focus on the results from the EDR3 in our study.

\subsection{Redshift}

We used the Optical Characteristics of Astrometric Radio Sources (OCARS; Malkin 2018) catalog to search for the redshifts. The OCARS catalog conveniently provides the redshifts for radio sources by collecting them in the literature. Among the 2460 sources, we obtained the redshifts for 2198 sources, or $\sim 89 \%$. They are in the range 0.01-5.06 with mean and median values of 1.28 and 1.18 , respectively.

\section{Results}

\subsection{Arc length $\rho$}

We examined the arc lengths $\rho$ between the VLBI and Gaia source position estimates with respect to the CARMS values. Table 1 shows the mean and median values of $\rho$ and $\sigma_{\rho}$ for different ranges of CARMS values. The median $\rho$ steadily increases from $\sim 0.4$ mas to $\sim 1.3$ mas when the CARMS values increase from 0.4 . The mean $\rho$ increases more significantly from $\sim 0.7$ mas to $\sim 3.7$ mas. The median $\rho$ begins to arise when $\mathrm{CARMS} \simeq 0.6$; the mean $\rho$ arises significantly, above 1.0 mas, when $\mathrm{CARMS} \simeq 0.3$. We note that in general a smaller CARMS value of a source indicates that it causes less significant structure effects.

When CARMS $<0.3$, the arc lengths $\rho$ have mean values of $\sim 0.7$ mas and median values of $\sim 0.4$ mas, and their uncertainties have mean values of $\sim 0.4$ and median values of $\sim 0.3$. It is reasonable to expect that these arc lengths will decrease with better uncertainties from Gaia in the near future, as happened from the DR2 to the EDR3. However, when CARMS $>0.6$, the arc lengths are larger and statistically significant, and they have even better uncertainties than the sources with CARMS $<0.3$. It is obvious that the sources with extended structure have larger position differences between VLBI and Gaia, which are statistically very significant, whereas the sources with minimum structure tend to have smaller position differences, which are statistically insignificant.

The mean $\rho$ is always larger than the median due to a small fraction of sources having considerably larger $\rho$ than the rest of sources in each group. The differences between the mean and median $\rho$ increase with the CARMS values. 

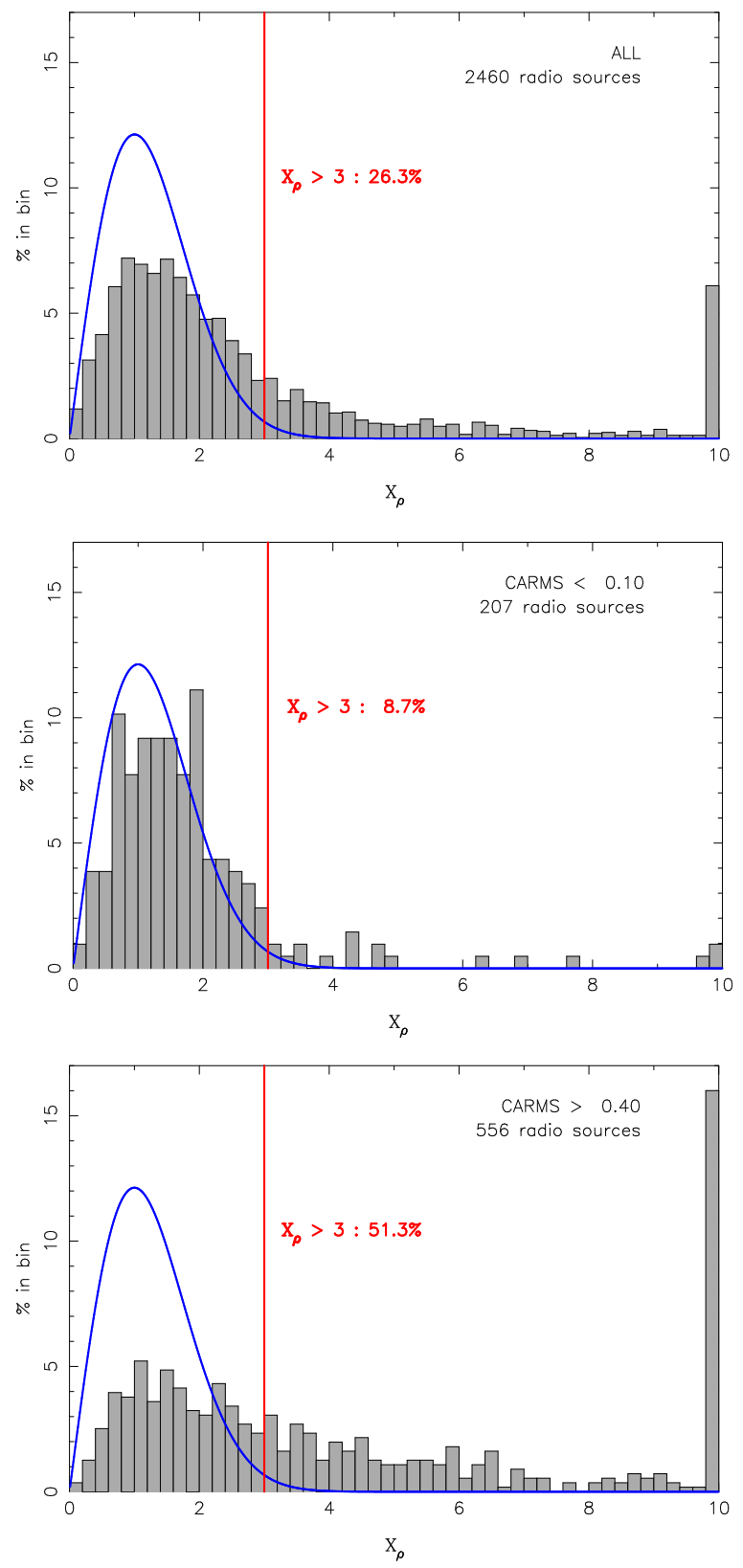

Fig. 1. Histogram of $X_{\rho}$ for the 2490 sources (top), the sources with CARMS $<0.10$ (middle), and the sources with CARMS $>0.40$ (bottom). The sources with $X_{\rho}>10$ are accounted for in the last bin. The blue curves show the Rayleigh distributions with unit standard deviation. The total number of sources in each of the three samples is shown in black in each panel (top right), and the number of the sources with $X_{\rho}>3.0$ is in red. The straight red lines correspond to $X_{\rho}=3$. The remarkable differences in the distributions of $X_{\rho}$ between these three groups of sources are the numbers of sources in the last bins, $X_{\rho}>9.8$.

\subsection{Normalized arc length $X_{\rho}$}

We examined the normalized arc lengths $X_{\rho}$ with respect to the CARMS values. The statistics of $X_{\rho}$ are shown in Table 1. A dependence of $X_{\rho}$ on the CARMS values is revealed. Figure 1 shows the three distributions of $X_{\rho}$ for the 2460 common sources (top), the sources with CARMS $<0.10$ (middle), and the sources with CARMS $>0.40$ (bottom). About $26 \%$ of the 2460 sources have $X_{\rho}>3$. For the 207 radio sources with little structure, the distribution of $X_{\rho}$ shown in the middle panel is close to the expected Rayleigh distribution; however, for the 556 radio

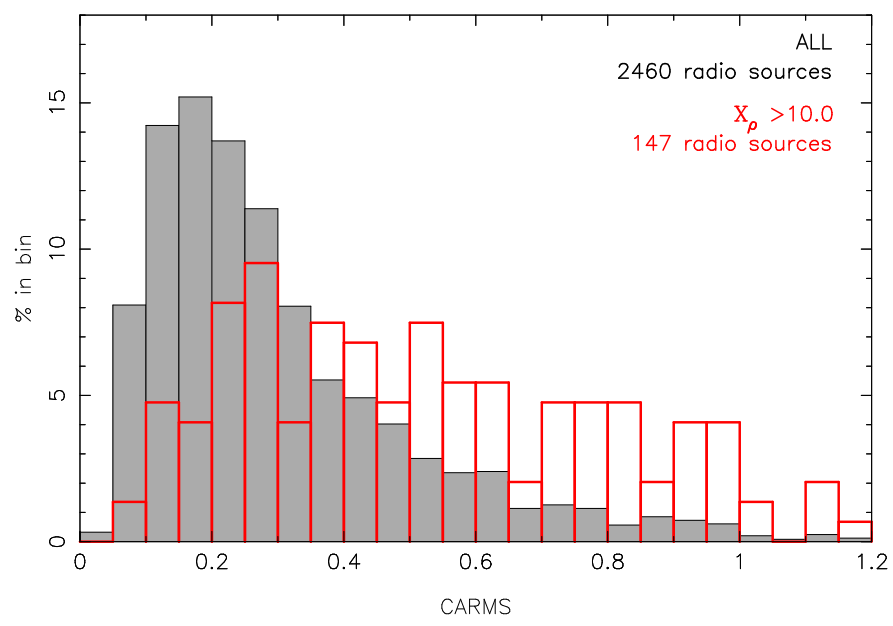

Fig. 2. Histogram of the CARMS values for the 2460 radio sources (filled gray bars) and for the 147 radio sources with $X_{\rho}>10.0$ (open red bars). About $3 / 5$ of these 147 sources have the CARMS values larger than 0.40 , while less than $1 / 4$ of the 2460 sources have the CARMS values larger than 0.40 .

sources with CARMS $>0.40$, the distribution of $X_{\rho}$ clearly deviates from the Rayleigh distribution (one-half of the sources have $X_{\rho}>3$, and one-sixth even have $X_{\rho}>10$ ). The probability of having statistically significant position differences is doubled for the radio sources with extended source structure (CARMS > 0.4).

Figure 2 shows the distributions of the CARMS values for all 2460 sources (gray filled bins) and the 147 sources with $X_{\rho}>10$ (red open bins). The mean and median CARMS values for all 2460 sources are 0.30 and 0.24 , respectively; for the 147 sources with $X_{\rho}>10$ these values are 0.52 and 0.48 . About $60 \%$ of the sources with $X_{\rho}>10$ have CARMS $>0.40$. Given that only $23 \%$ of the 2460 sources have CARMS $>0.40$, the high correlation is also identified between the sources with statistically significant position differences and the sources with extended structure.

The differences in the CARMS values for the sources with various ranges of $\rho$ and $X_{\rho}$ are shown in Table 2. For different magnitudes of $\rho$ the mean and median CARMS values for the sources with $X_{\rho}>4$ are all the largest among the three categories based on $X_{\rho}$; these values for the sources with $X_{\rho}$ in the range of 3-4 are larger than for the sources with $X_{\rho}<3$. On average, the difference in the CARMS values is $\sim 0.2$ between the sources with and without statistically significant position differences. There is only a slight increase in the mean and median CARMS values as $\rho$ increases for $X_{\rho}>4$. We recommend caution when interpreting the results in Table 2, because the values will change with better uncertainties of source positions in the future Gaia data releases. With the significant improvement in position uncertainties expected from the Gaia observations, the sources with current $X_{\rho} \leq 3$ can have $X_{\rho}>3$, as happened for the Gaia DR2 compared to the Gaia DR1 and for Gaia EDR3 compared to the Gaia DR2. Meanwhile, the arc lengths $\rho$ for the 1813 sources with $X_{\rho} \leq 3$ will generally decrease, which can be demonstrated by the Gaia DR2 and EDR3. The arc lengths of these 1813 sources are all smaller than 4.0 mas; the number of the sources with $\rho \geq 4.0$ mas should not be changed dramatically, unless a few new common sources between Gaia and the ICRF3 can be identified from the future Gaia data releases. Since the ICRF3 sources were systematically included in the Gaia quasar list, those missing sources in the Gaia EDR3 are 
Table 2. CARMS values with respect to $\rho$ and $X_{\rho}$.

\begin{tabular}{|c|c|c|c|c|c|c|c|c|c|}
\hline \multirow[t]{2}{*}{$\rho$ [mas] } & \multicolumn{3}{|c|}{ if $\left(X_{\rho}>4\right)$} & \multicolumn{3}{|c|}{ if $\left(4 \geq X_{\rho}>3\right)$} & \multicolumn{3}{|c|}{ if $\left(X_{\rho} \leq 3\right)$} \\
\hline & $N_{\text {src }}$ & Mean & Median & $N_{\text {src }}$ & Mean & Median & $N_{\mathrm{src}}$ & Mean & Median \\
\hline$<0.4$ & 34 & 0.43 & 0.44 & 33 & 0.35 & 0.30 & 1011 & 0.26 & 0.23 \\
\hline$[0.4-0.7)$ & 56 & 0.43 & 0.36 & 62 & 0.33 & 0.28 & 425 & 0.25 & 0.21 \\
\hline$[0.7-1.0)$ & 55 & 0.47 & 0.45 & 48 & 0.25 & 0.21 & 179 & 0.27 & 0.21 \\
\hline$[1.0-2.0)$ & 114 & 0.50 & 0.43 & 52 & 0.30 & 0.19 & 164 & 0.25 & 0.20 \\
\hline$[2.0-4.0)$ & 98 & 0.40 & 0.35 & 16 & 0.50 & 0.43 & 34 & 0.26 & 0.20 \\
\hline$[4.0-7.0)$ & 44 & 0.46 & 0.44 & 4 & 0.36 & 0.33 & 0 & $\ldots$ & $\ldots$ \\
\hline$\geq 7.0$ & 31 & 0.51 & 0.47 & 0 & $\ldots$ & $\ldots$ & 0 & $\ldots$ & $\ldots$ \\
\hline All & 432 & 0.46 & 0.42 & 215 & 0.32 & 0.27 & 1813 & 0.26 & 0.22 \\
\hline
\end{tabular}

Table 3. Optical $G$ magnitude and redshift $z$.

\begin{tabular}{lccccccc}
\hline \hline CARMS & \multicolumn{3}{c}{ Optical $G$ magnitude $[\mathrm{mag}]$} & & \multicolumn{3}{c}{ Redshift $z$} \\
\cline { 2 - 3 } \cline { 6 - 8 } & $N_{\text {src }}$ & Mean & Median & & $N_{z}$ & Mean & Median \\
\hline$<0.1$ & 207 & 19.283 & 19.486 & & 181 & 1.270 & 1.062 \\
{$[0.1-0.2)$} & 724 & 18.965 & 19.112 & & 624 & 1.188 & 1.072 \\
{$[0.2-0.3)$} & 617 & 18.665 & 18.759 & & 553 & 1.214 & 1.139 \\
{$[0.3-0.4)$} & 334 & 18.712 & 18.841 & & 305 & 1.355 & 1.292 \\
{$[0.4-0.5)$} & 220 & 18.460 & 18.498 & & 204 & 1.350 & 1.256 \\
{$[0.5-0.6)$} & 128 & 18.489 & 18.581 & & 116 & 1.413 & 1.312 \\
{$[0.6-0.7)$} & 87 & 18.381 & 18.474 & & 78 & 1.268 & 1.203 \\
{$[0.7-0.8)$} & 59 & 18.442 & 18.446 & & 53 & 1.622 & 1.400 \\
{$[0.8-0.9)$} & 35 & 18.678 & 18.740 & & 33 & 1.506 & 1.460 \\
$\geq 0.9$ & 49 & 18.359 & 18.597 & & 47 & 1.434 & 1.351 \\
\hline All & 2460 & 18.763 & 18.910 & & 2198 & 1.275 & 1.182 \\
\hline
\end{tabular}

probably too faint in the optical, and finding significantly more matches from Gaia is unlikely. As shown in Table 1, the uncertainties of $\rho$ have mean and median values of about 0.3 mas and 0.2 mas, which allow the large $\rho$ (e.g., $>4.7$ mas) to be confidently detected but are not able to fully identify the sources with $\rho<1.0$ mas. Therefore, when the final Gaia data release is available to identify more sources with small $\rho$ and large $X_{\rho}$, the mean and median CARMS values will thus decrease for the sources with $\rho<1.0$ mas and $X_{\rho}>4$. We expect to see the CARMS values steadily increasing with respect to $\rho$ in the future Gaia data releases, as we see that $\rho$ increases with CARMS in Table 1. In the following investigation we set the limit of $X_{\rho}=4.0$, at the $99.994 \%$ confidence level, to identify the sources with statistically significant position differences.

\subsection{Optical $G$ magnitude and redshift}

We examined optical $G$ magnitude and redshift $z$ with respect to the CARMS values to investigate whether there is any potential correlation between the CARMS values and the optical properties. Table 3 shows the statistics of $G$ and $z$. The mean and median magnitudes both generally decrease with respect to the CARMS values; the difference in $G$ between the sources with CARMS $<0.1$ and with CARMS $>0.9$ is about 0.9 mag. Based on the high correlation between the radio luminosity and the optical luminosity shown in Arshakian et al. (2010), the sources with higher luminosity at optical wavelengths will have higher radio flux densities. We can also expect a correlation between radio luminosity and extended structure which is driven by jet power: higher power means higher radio luminosity and more extended structure in linear scale due to the jet being able to drill its path. Radio sources with higher flux densities tend to have more extended structure and consequently larger CARMS values, as shown for the 30 most frequently observed sources in geodetic VLBI by Xu et al. (2019). Since the CRF sources are flux-limited, at high redshifts the sources must have high luminosity, and consequently their powers and extents are larger than at low redshift. This should partly explain the correlation between $z$ and CARMS in the table. The correlation between CARMS and both $G$ and $z$ seems to be significant.

We examined $G$ and $z$ in more detail. This investigation can be biased because the uncertainties of Gaia positions depend on $G$, as shown in Gaia Collaboration (2018b). The statistics of arc lengths and normalized arc lengths with respect to $G$ can be dramatically changed when new position estimates with improved uncertainties are available from Gaia in the near future. We nevertheless attempt to address it based on the Gaia EDR3.

Table 4 shows the statistics of arc lengths, the major axes of the error ellipses of the Gaia positions and the VLBI positions, the CARMS values, and $z$ with respect to different optical $G$ magnitudes for the 2028 sources with $X_{\rho} \leq 4$. As we expect, the values of $G$ and $z$ are positively correlated for these sources: when an object is farther away it appears dimmer. The differences of the mean CARMS values at various ranges of $G$ are no larger than 0.06 , and those of the median values are no larger than 0.08. There is a small decrease in the CARMS values when $G$ increases, which demonstrates that when a source locates farther away the scale of its structure may decrease. The 
Table 4. Statistics of the 2028 sources with $X_{\rho} \leq 4$.

\begin{tabular}{|c|c|c|c|c|c|c|c|c|c|c|}
\hline \multirow[t]{2}{*}{$G[\mathrm{mag}]$} & \multirow[t]{2}{*}{$N_{\text {src }}$} & \multicolumn{2}{|c|}{$\rho$ [mas] } & \multicolumn{2}{|c|}{$\sigma_{\text {pos, } \max }[\mathrm{mas}]$} & \multicolumn{2}{|c|}{ CARMS } & \multicolumn{3}{|c|}{$z$} \\
\hline & & Mean & Median & Gaia & VLBI & Mean & Median & $N_{z}$ & Mean & Median \\
\hline$<15.0$ & 7 & 0.304 & & 0.020 & ( 210 & & & 7 & 0.304 & 0.200 \\
\hline $15.0-16.0)$ & 35 & 258 & 75 & 030 & 5 & 30 & 0.2 & 35 & 59 & 0.310 \\
\hline & 29 & & & & & & & 29 & & 0.557 \\
\hline 16.5-17.0) & 62 & 0.283 & & & & & 0.24 & 59 & & 1.003 \\
\hline$[17.0-17.5)$ & 125 & 0.337 & 0.271 & 0.072 & 0.199 & 0.29 & 0.2 & 120 & 1.029 & 0.954 \\
\hline$[17.5-18.0)$ & 176 & 0.312 & & 0.094 & & & 0.2 & 172 & 1.211 & 1.093 \\
\hline-18.5$)$ & 265 & 0.347 & 288 & 126 & 210 & 28 & 0.24 & 251 & 1.261 & 1.200 \\
\hline$[18.5-19.0)$ & 312 & 0.392 & & 0.179 & 0.207 & 0.27 & 0.23 & 292 & 1.452 & 1.384 \\
\hline [19.0-19.5) & 331 & 0.492 & 0.399 & 0.247 & 0.230 & 0.25 & 0.21 & 301 & 1.423 & 1.375 \\
\hline & 315 & 0.611 & 0.491 & 0.370 & 0.223 & 0.24 & 0.19 & 259 & 1.428 & 1.300 \\
\hline$[20.0-20.5)$ & 275 & 0.917 & 0.790 & 0.623 & 0.228 & 0.24 & 0.19 & 202 & 1.502 & 1.375 \\
\hline$\geq 20.5$ & 96 & 1.687 & 1.434 & 1.079 & 0.272 & 0.27 & 0.20 & 64 & 1.274 & 0.980 \\
\hline All & 2028 & 0.633 & 0.454 & 0.293 & 0.216 & 0.26 & 0.22 & 1791 & 1.314 & 1.219 \\
\hline
\end{tabular}

Notes. The values in the fifth and sixth columns are the mean $\sigma_{\text {pos, max }}$ for Gaia and VLBI position estimates, respectively.

Table 5. Statistics of the 432 sources with $X_{\rho}>4$.

\begin{tabular}{|c|c|c|c|c|c|c|c|c|c|c|}
\hline \multirow[t]{2}{*}{$G[\mathrm{mag}]$} & \multirow[t]{2}{*}{$N_{\text {src }}$} & \multicolumn{2}{|c|}{$\rho$ [mas] } & \multicolumn{2}{|c|}{$\sigma_{\text {pos,max }}[\mathrm{mas}]$} & \multicolumn{2}{|c|}{ CARMS } & \multicolumn{3}{|c|}{$z$} \\
\hline & & Mean & Median & Gaia & VLBI & Mean & Median & $N_{z}$ & Mean & Median \\
\hline$<15.0$ & 9 & 0.369 & & .016 & & & 0.30 & 9 & 0.228 & 0.160 \\
\hline $.0-16.0)$ & 17 & & & 32 & & & & 17 & & 0.302 \\
\hline [16.0-16.5) & 19 & 1.083 & 0.839 & .040 & 0.121 & 0.56 & 0.60 & 18 & 1.182 & 1.258 \\
\hline$[16.5-17.0)$ & 34 & 3.820 & 0.867 & .062 & 0.1 & & & 34 & & 1.140 \\
\hline & 43 & 1.810 & & 8 & 0.1 & & 0. & 43 & 1.0 & 0.9 \\
\hline$[17.5-18.0)$ & 59 & 1.581 & 0.951 & 0.098 & 0.157 & 0.47 & 0.44 & 57 & 1.283 & 1.285 \\
\hline [18.0-18.5) & 68 & 2.483 & 1.505 & 0.146 & 0.167 & 0.44 & 0.39 & 68 & 1.228 & 1.208 \\
\hline$[18.5-19.0)$ & 52 & 4.258 & 1.535 & 0.184 & 0.229 & 0.44 & 0.41 & 48 & 1.065 & 0.949 \\
\hline [19.0-19.5) & 53 & & 2.289 & 0.250 & 0.222 & 0.46 & 0.41 & 44 & 1.061 & 0.726 \\
\hline$[19.5-20.0)$ & 35 & 4.953 & 2.987 & 0.405 & 0.241 & 0.44 & 0.35 & 31 & 1.054 & 0.667 \\
\hline$[20.0-20.5)$ & 28 & 5.344 & 3.623 & 0.622 & 0.255 & 0.31 & 0.30 & 26 & 0.940 & 0.770 \\
\hline$\geq 20.5$ & 15 & 4.176 & 3.151 & 0.805 & 0.292 & 0.49 & 0.46 & 12 & 1.254 & 1.037 \\
\hline All & 432 & 3.173 & 1.510 & 0.207 & 0.183 & 0.46 & 0.42 & 407 & 1.103 & 0.901 \\
\hline
\end{tabular}

Notes. The values in the fifth and sixth columns are the mean $\sigma_{\text {pos, max }}$ for Gaia and VLBI position estimates, respectively.

magnitudes of $\rho$ gradually increase with respect to $G$; however, the position uncertainties of both Gaia and VLBI also vastly increase. Since the ratio of the arc lengths to its uncertainties is always at the same level for different ranges of $G$, it is not possible from the result to conclude that there is dependence of $\rho$ on $G$.

Table 5 shows the statistics of the same quantities as Table 4 but for the 432 sources with $X_{\rho}>4$. The arc lengths increase by a factor of $\sim 10$ from $G<15 \mathrm{mag}$ to $G \geq 20 \mathrm{mag}$. This apparent dependence of $\rho$ on $G$, however, is mainly due to the high correlation between the Gaia position uncertainties and $G$, as shown in the table. Because the Gaia position uncertainties get dramatically worse as $G$ becomes higher, a uniformed threshold of $X_{\rho}$, which is 4 in the study, forces only the sources with large enough arc lengths to be selected at the higher optical magnitudes. As discussed before, these statistics will change when new position estimates are available from the future Gaia data releases.

By comparing the results in Tables 4 and 5, the major differences of these two groups of sources are found to be CARMS and $z$. The CARMS values of the sources with $X_{\rho}>4$ are larger (by $~ 0.2$ ) than those of the sources with $X_{\rho} \leq 4$; the mean and median $z$ values are smaller (by 0.21 and 0.32 , respectively). The relationship between $G$ and $z$ for these two groups of sources are shown in Fig. 3. The sources with $X_{\rho} \leq 4$ have $z$ steadily increasing over $G$, while the sources with $X_{\rho}>4$ even show a small decrease in $z$ when $G>16.5$ mag.

We argue that the statistically significant position differences may also be associated with, for instance, some weak but nearby (small z) optical objects.

\section{Discussion}

\subsection{Radio source structure}

The CRF sources have radio emission with angular scales at milliarcsecond levels over the sky, called source structure. It causes structure delays of up to hundreds of picoseconds, as shown in modeling by Charlot (1990b) and in actual observations by Xu et al. (2016). Based on the CONT14 observations ${ }^{9}$,

9 https://ivscc.gsfc.nasa.gov/program/cont 14/ 


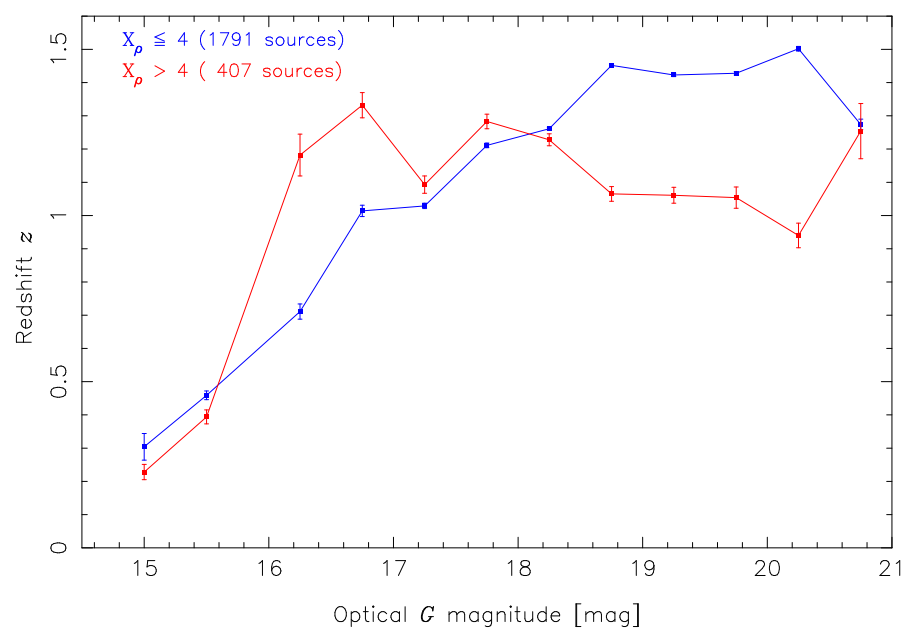

Fig. 3. Mean redshift values with respect to the optical $G$ magnitudes for the 2198 sources with available redshifts. The blue curve is for the sources with $X_{\rho} \leq 4$, and the red curve is for the sources with $X_{\rho}>4$. The error bars show the estimated uncertainties of the mean values. The bin windows of $G$ are shown in the first column in Table 4. The sources with $X_{\rho}>4$ have substantially lower $Z$ at $G>18.5 \mathrm{mag}$, but higher $z$ at $G \simeq 16.5$ mag than the sources with $X_{\rho} \leq 4$. The statistics are shown in Tables 4 and 5 .

Anderson \& Xu (2018) suggested that source structure is the major contributor to errors in the astrometric and geodetic VLBI. Since these effects in VLBI group delays have not been modeled in the VLBI data analysis, based on which the ICRFs were built and maintained, the source positions from VLBI change over time due to both the different observing geometry between antennas and sources and the varying structure. For a large fraction of CRF sources, the structure effects can change their positions at the level of 0.5 mas, as shown in the position time series of 39 well-observed sources (Ma et al. 2009, see the plots in the IERS Technical Note $35^{10}$ ). The number of sources affected by the structure effects dramatically increases when we consider the position differences between Gaia and VLBI down to the level of $\sim 0.3$ mas. Based on the CARMS values, $40 \%$ of CRF sources have significant structure.

CARMS tells the structure effects in amplitude observables. For a source with CARMS $=0.1$, the ratios of the amplitude observables to the various combinations of quadrangle have an rms of 1.1. Those ratios have an rms of 1.5 for CARMS $=0.4$, and 1.8 for CARMS $=0.6$. It is straightforward to understand that sources with a small CARMS value are close to point-like, and those with a large CARMS value have extended structure.

In Fig. 4, we show the images from MOJAVE for four sources, 0048-097, 0059+581, 1803+784, and 1928+738. Since the VLBI observations for deriving the images are at different frequencies by different antenna arrays during different time periods compared to the observations for the ICRF3 and the CARMS values, we cannot expect an exact proportional relation between the CARMS values and the scales of the MOJAVE images. However, they are already of great help to demonstrate the differences between the CARMS values smaller and larger than 0.3. The two sources 0048-097 (CARMS $=0.11)$ and $0059+581(\mathrm{CARMS}=0.27)$ only have compact cores, whereas the other two sources, $1803+784(\mathrm{CARMS}=0.35)$ and

\footnotetext{
10 https://www.iers.org/SharedDocs/Publikationen/EN/ IERS/Publications/tn/TechnNote35/tn35_017.pdf?__blob= publicationFile\&v $=1$
}

$1928+738($ CARMS $=0.88)$, have significant emissions from the jets on milliarcsecond scales. The relative positions between Gaia and VLBI are also shown in the plots. It is obvious in the plots that the Gaia-VLBI position differences are typically parallel to the jet directions, which has already been reported by Kovalev et al. (2017) and Petrov et al. (2019) and will be discussed in Sect. 4.5.

There are four remarks concerning CARMS. First, it was calculated based on actual VLBI observations rather than the maps of radio sources. Once the CARMS is large, the source should have extended structure; however, if the source has extended structure, it does not necessarily have a large CARMS value due to insufficient observations in terms of $(u, v)$ coverage to capture the structure. However, the great advantage of using actual VLBI observations is that it quantifies the magnitude of structure effects over the whole time period of 40 years. Second, CARMS is based on (log) closure amplitudes, which are not sensitive to the absolute source position. Therefore, only the relative structure (i.e., the relative positions and the relative fluxes between the multiple components) is defined by CARMS; if a source with compact structure changes its position on the sky, the CARMS value cannot predict that change. Third, since there was no attempt to do proper weighting for different sizes of quadrangle and select an independent set of closure amplitudes for each individual source in deriving CARMS values, it becomes difficult to determine a source with a medium CARMS value, $0.25-0.30$, as having structure and to what extent. Fourth, CARMS was derived from the $X$-band observations only, while the ICRFs are based on the ionosphere-free delays through the linear combination of the group delays at the $S / X$ band. The structure effects in the $S$-band observations thus are ignored in this study. Even though the contribution of the structure effects at the $S$ band is scaled down by a factor of $\sim 13.8$ in that linear combination process, it can be significant for some radio sources. This should partly explain why there are sources with CARMS $<0.10$ but with $X_{\rho}>3.0$, as shown in the middle panel of Fig. 1.

Modeling structure effects is still missing in astrometric and geodetic VLBI data analysis, even after several decades of discussion. The practical problems are to continuously make images for hundreds of sources and for each source many times if the structure changes. The main challenge is that the images for modeling structure effects have to be registered over time for each source in order to maintain a stable CRF at high accuracy. The next generation of geodetic VLBI, known as the VLBI Global Observing System (VGOS) (Niell et al. 2007; Petrachenko et al. 2009), requires the images of each source at the four different bands to be registered in the range of 3.014.0 GHz (Xu et al. 2021b). Otherwise, only the relative structure effects can be reduced, and the misalignment of the images at different epochs or at different frequency bands due to core shift, discussed in the next section, inevitably leads to source position variations. Due to the limitation in imaging resolutions, identifying the reference points in structure and images is difficult for accuracy levels better than 0.1 mas. Therefore, aligning the images and investigating core shift are crucial in order to mitigate these systematic effects.

\subsection{Core shift}

Source structure is frequency-dependent due to two factors: (1) the steep spectrum of the extended jet causing the sources to have larger scales at lower frequencies; and (2) synchrotron self-absorption causing changes in the optical depth along the 

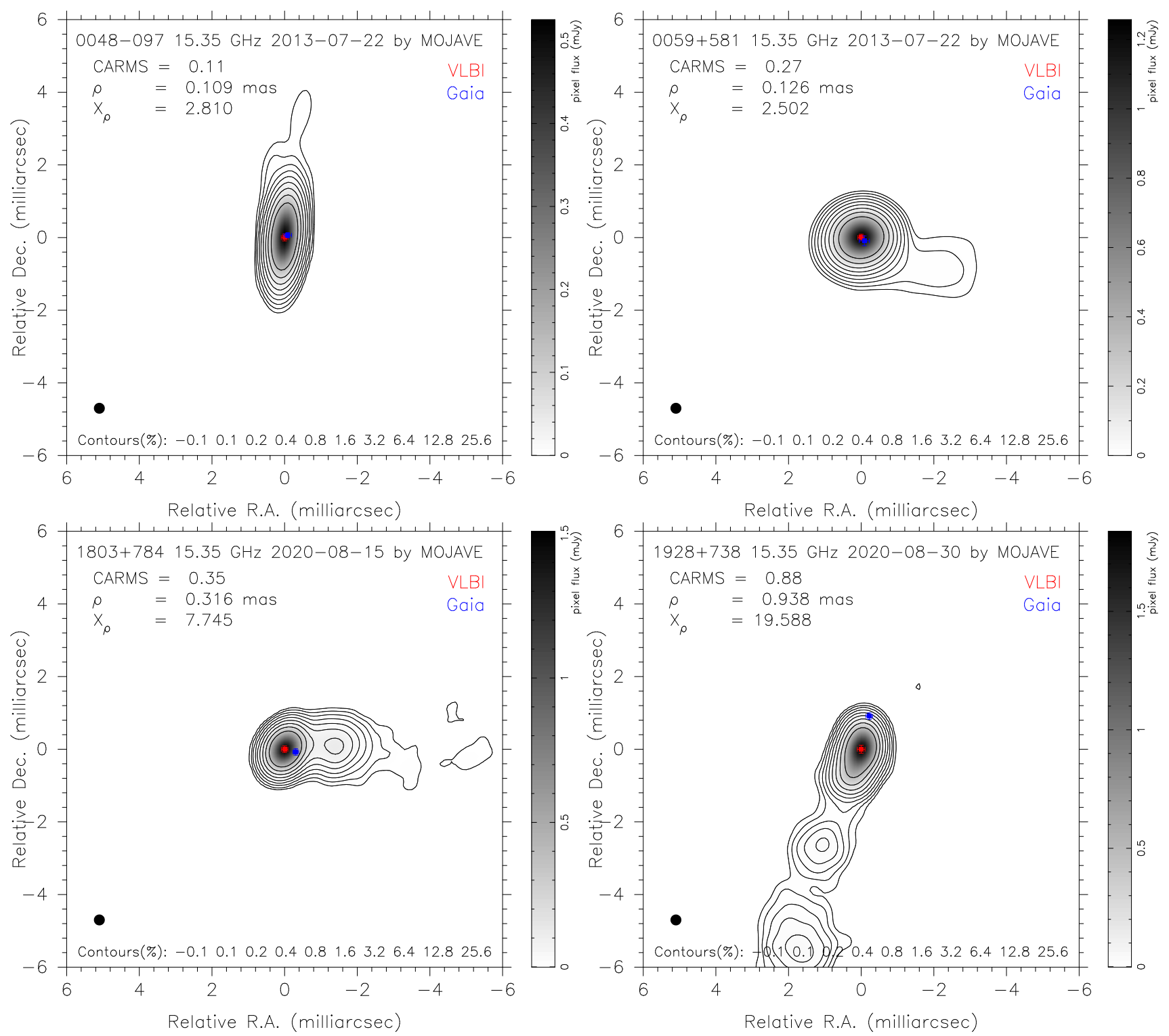

Fig. 4. MOJAVE images of four sources, 0048-097 (CARMS $=0.11$, upper left), 0059+581 (CARMS $=0.27$, upper right), 1803+784 $($ CARMS $=0.35$, bottom left $)$, and 1928+738 (CARMS $=0.88$, bottom right $)$. These images were made based on VLBA observations at $15.35 \mathrm{GHz}$ by the MOJAVE project. The peaks of flux are selected as the origins. The VLBI positions are formally assumed to be at the origins, as shown by the red dots. Since the MOJAVE images and the ICRF3 are derived from observations at different frequencies, this assumption may introduce systematic errors. Based on their position differences between the Gaia EDR3 and the ICRF3, the Gaia positions are thus located at the blue dots. The error bars are the $3 \sigma$ uncertainties of right ascension and declination from Gaia and VLBI. It is also conspicuous that when $X_{\rho}>4$ the VLBI to Gaia position vectors favor the directions along and opposite to the jets, as shown by Kovalev et al. (2017), Petrov et al. (2019). The $\rho$ and $X_{\rho}$ values are shown in the upper left corner of each plot. These four plots demonstrate how the scales of the structure appear in terms of different CARMS values. Nevertheless, we should mention that the CARMS values and the ICRF3 are based on VLBI observations at the frequency band around $8.4 \mathrm{GHz}$ over 40 years, while these images were made from observations at $15.35 \mathrm{GHz}$ during the short periods shown at the top of each plot. The jet components always become more prominent at the lower frequency bands. The images were convolved with a circular beam of 0.3 mas as indicated by the black circle in the bottom left corner, about $40 \%$ of the typical MOJAVE beam size. Overlay contours are shown at ten levels of peak percentage specified in the bottom of plots.

jet. The latter factor leads to changes in the position of the core, where the optical depth is unity, depending on the observing frequency. This effect, called core shift, was predicted by Blandford \& Königl (1979). When the observing frequency increases, it causes the position of the core to move towards the jet base.

Core shift was first measured for the source $1038+528 \mathrm{~A}$ with a magnitude of $\sim 0.7$ mas at $2.3 \mathrm{GHz}$ and $8.4 \mathrm{GHz}$ by referring to its nearby source $1038+528 B$ (Marcaide et al. 1985). Since then it has been measured for 29 sources with a median value of 0.44 mas between $2.3 \mathrm{GHz}$ and $8.4 \mathrm{GHz}$ by Kovalev et al. (2008), for 20 sources with a median value of 1.21 mas between 1.4. GHz and $15.4 \mathrm{GHz}$ and 0.24 mas between $5.0 \mathrm{GHz}$ and $15.4 \mathrm{GHz}$ by Sokolovsky et al. (2011), for 163 sources with a median value of 0.128 mas between $8.4 \mathrm{GHz}$ and $15 \mathrm{GHz}$ by Pushkarev et al. (2012), and for 40 sources with a typical value of 0.5 mas between $2.3 \mathrm{GHz}$ and $8.4 \mathrm{GHz}$ by Plavin et al. (2019b). The frequency-dependency of the core position can be parameterized by $k v^{-\beta}$, where $k$ is a source-dependence core shift parameter (it can be variable over time according to the 
study of Plavin et al. 2019b), $v$ is the observing frequency, and $\beta$ is an astrophysical parameter. So far, $\beta$ has been measured to be close to 1 (Lobanov 1998; Sokolovsky et al. 2011), which agrees with the prediction under the condition of the equipartition between jet particle and magnetic field energy densities (Blandford \& Königl 1979).

The impact of core shift on astrometric positions measured by VLBI was discussed by Porcas (2009) using a simple model of a point-source core. Based on the median core shift between $2.3 \mathrm{GHz}$ and $8.4 \mathrm{GHz}$ of 0.44 mas (Kovalev et al. 2008), the core position is shifted by 0.166 mas at a frequency of $8.4 \mathrm{GHz}$, and varies by 0.014 mas over the frequency band of $8.2-8.9 \mathrm{GHz}$ used in most of geodetic VLBI observations. The position shift of 0.166 mas can cause visibility phase variations of several degrees over the band, which are canceled out exactly by the additional phase variations due to the position shifts of 0.014 mas over the band. It was shown that given $\beta=1$, group delays of observations on a point-like source refer to a fixed point at the jet base at any frequency and at any time, whether $k$ varies or not over time. It is therefore believed that core shift will not contribute to the position differences between Gaia and VLBI, given that $\beta \simeq 1$.

Our special concern about core shift is not only the robust validation of $\beta \simeq 1$ for the CRF sources, but also the simple source model used in Porcas (2009). Core shift has two effects on source structure: (1) moving the absolute position of the core towards the jet base when the frequency increases; and (2) changing the relative positions between the core and the jet components in structure. Apparently, the discussion of Porcas (2009) investigated the first effect only. The truth is again that almost all the CRF sources have structure on milliarcsecond scales, which changes over time. In the previous discussion, the relative positions between the core and the jets will also be changed by 0.014 mas over the band to the opposite direction of the absolute position shift of the core. The cancellation of the acrossband phase variations in the point-source case breaks down for extended sources. Therefore, core shift can influence the position estimates determined from VLBI group delays. In this context, even though there may be no real connection between the magnitude of core shift and the scales of source structure, the impact of core shift will correlate with structure effects; extended sources with large source structure effects tend to have larger core shift effect in the position differences between radio and optical than the sources with minimum structure. Further studies are needed to verify this assumption.

\subsection{Sources with $\rho>4.0$ mas and $X_{\rho}>4$}

There are 75 sources with $\rho>4.0$ mas and $X_{\rho}>4$. Among them, 53 sources have CARMS $>0.3$ and 41 sources have CARMS $>0.4$. Of the 22 sources with CARMS values $\leq 0.3,20$ sources have available $z$ values, and 15 sources have $z<0.7$. The median $z$ of these 20 sources is 0.25 , which is only one-fifth of the median $z$ of the 2198 sources with known $z$. A small fraction of these sources seem to be weak but nearby optical objects. It is important to investigate this further.

\subsection{Magnitudes of the position differences}

With an improvement in Gaia position estimates in the near future, the number of the sources with $X_{\rho}>4$ may continue to increase. However, there should be no significant increase in the number of sources with extremely large differences (e.g., $\rho>4.0$ mas); currently, there are 79 sources, less than $4 \%$.
As shown in Tables 4 and 5, there are 615 sources with $G<$ $18 \mathrm{mag}$, and the mean semi-major axis of the error ellipses of the Gaia positions for these 615 sources is already smaller than 0.1 mas. In this sample of 615 sources, 181 sources have $X_{\rho}>4$, or $40 \%$. About $74 \%$ of these 181 sources have $\rho<1.5$ mas; the median $\rho$ is $\sim 0.8$ mas. Therefore, the magnitude of $\rho$ for the majority of the sources with $X_{\rho}>4$ is expected to be at the same level as source structure effects and core shift. For the 434 sources with $X_{\rho} \leq 4$, the median $\rho$ is $\sim 0.24$ mas, which is at the same level as their uncertainties dominated by VLBI. This may provide insights into the final agreement of source positions between Gaia and VLBI for the whole ensemble of common sources.

If we assume that the median uncertainty of the Gaia source positions at higher optical magnitudes is $\sim 0.1$ mas, which is better than the predicted end-of-mission accuracies but still possible (Perryman et al. 2001; de Bruijne et al. 2014), the Gaia and VLBI positions will agree with each other within their uncertainties for the $3 / 5$ sources, and the median $\rho$ for these sources will be at the level of 0.24 mas. There will be $2 / 5$ sources having statistically significant position differences with a median $\rho$ of 0.8 mas.

Based on about 2000 evenly distributed sources over the sky with position differences of $\sim 0.24$ mas, the orientation stability of the Gaia frame with respect to the ICRF3 may be achieved at the level of ten microarcseconds ( $\mu \mathrm{as}$ ); it is sufficient to detect systematic position differences between Gaia and VLBI at the level of hundreds of $\mu$ as. Several hundreds of sources with welldetected position differences at such levels will provide invaluable information to investigate the physical properties of radio sources.

\subsection{Directions of the position differences}

Source structure and core shift are expected to cause the derived source positions from VLBI to shift towards the jets. If the VLBI-to-Gaia position vectors are opposite to the directions of the radio jets, as shown for the source $1928+738$ in the bottom right panel of Fig. 4, the position differences can be explained by source structure effects or core shift. However, it is difficult to explain these position vectors along the jets, as shown for the source $1803+784$ in the bottom left panel, as the effects of radio source structure and core shift. Recent studies have demonstrated that the VLBI-to-Gaia position vectors favor the directions both along and opposite to the jets (Kovalev et al. 2017; Petrov et al. 2019), and more sources have these position vectors along the jets than opposite to the jets. The presence of parsecscale optical jet structure in the directions of the radio jets is one proposed explanation of the phenomenon in these studies.

We compared the directions of the VLBI-to-Gaia position vectors and of the radio jets based on the MOJAVE data. The jet directions were calculated as the median values of the jet position angles for the multiple jets of each individual source in the MOJAVE project. These jet position angles were robustly determined from multiple-epoch measurements by MOJAVE (Lister et al. 2018). Figure 5 shows the 208 sources with the uncertainties of both the jet position angles and the VLBI-to-Gaia position directions smaller than 30 degrees as gray dots and the 81 sources with those uncertainties smaller than 12 degrees as red dots. About $88 \%$ of these 81 sources have the VLBI-to-Gaia position vectors parallel to the jet directions within 25 degrees and $96 \%$ within 45 degrees. It enhances the already known results from Kovalev et al. (2017) and Petrov et al. (2019) with stronger evidence. The majority 


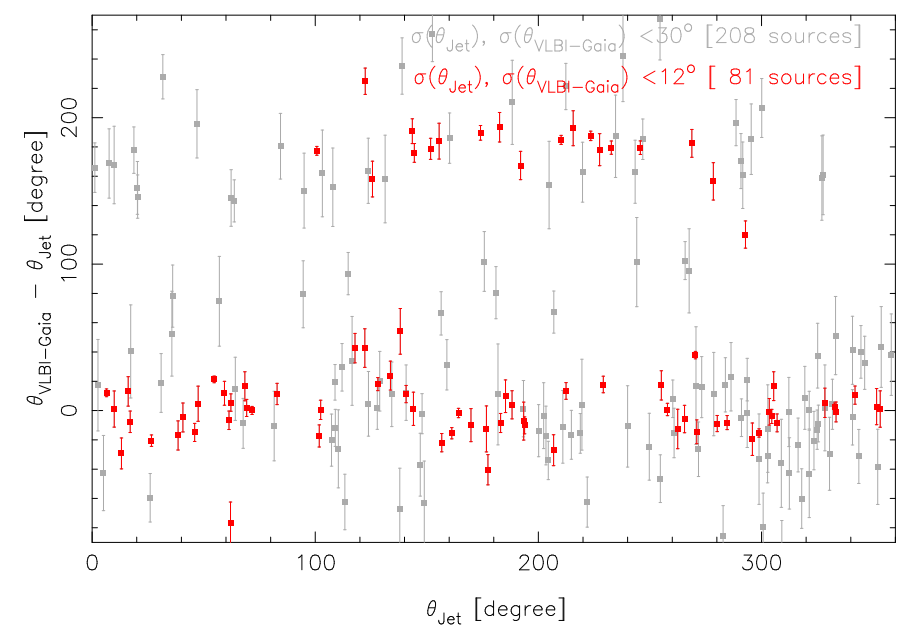

Fig. 5. Angles of the VLBI-to-Gaia position vectors with respect to the jet directions as a function of the jet position angles based on the MOJAVE data. The error bars shown are the combined uncertainties from the formal errors of the two directions. There are 327 sources with robust multi-epoch and multi-jet position angles, cross-matched from the 3142 sources. Of these, 208 sources have both the uncertainties of the VLBI-to-Gaia position directions and the median jet directions smaller than 30 degrees, and are shown as gray dots. There are 81 sources with those uncertainties smaller than 12 degrees, shown as red dots. For these 81 sources, the median $\rho$ is 0.93 mas, and the $X_{\rho}$ values are larger than 3.3. Among them, 54 sources have the directions of the position differences along the jet directions within 25 degrees and their $\rho$ are in the range $0.2-28.0$ mas; 17 sources have the directions of the position differences opposite to the jet directions, and their $\rho$ values are in the range 0.2-39.1 mas.

of the sources have the directions of the position vectors along the jets and a significant fraction of sources have those vectors opposite to the jets, also confirmed by this small sample of welldetermined jet position angles.

However, we address several cases where the jet position angles can be determined in the opposite direction. Figure 6 shows the images of source 0743-006, one of the ICRF3 defining sources but with the CARMS value of 0.64, at two different epochs. It has two compact components separated by $\sim 1$ mas and a fuzzy emission region extending to the northeast. The peak of flux changed between the two components from 2010 to 2020. According to its jet motions from model fitting, which are relatively small and weak for this particular source, the core was suggested by MOJAVE to be the peak of flux in the image from 2010, and consequently it has two-sided jets. It seems that the southwest component can be the core, which means that the source actually has a one-sided jet. In this case, the jet position angle can be determined with an offset of 180 degrees. The source has $\rho=1.1$ mas and $X_{\rho}=16.5$. If the southwest component is the core, the difference between its Gaia and VLBI positions can be explained by its radio source structure. As we can see, in the right-hand plot, if we move the VLBI position to the next component to the upper left, then the Gaia position fits the core very well.

We further discuss two cases, sources 0923+392 and $0429+415$, of extremely large and statistically significant position differences between VLBI and Gaia, which can be explained by their radio structure. Their MOJAVE images are shown in Fig. 7 with their relative positions between VLBI and Gaia illustrated. The figure demonstrates that the source positions from geodetic VLBI are dominated by the positions of the peak fluxes, whereas the optical positions from Gaia are located close to the cores. The separations between the cores and the jets for the CRF sources are typically at the milliarcsecond level, as shown in Figs. 4 and 6, and up to tens of milliarcseconds, as shown in Fig. 7. It should be noted that for a significant number of sources the VLBI position seems to be that of a jet component rather than the core. Without absolute position information in the MOJAVE images, however, we have no knowledge of where the VLBI position really is. Since the VLBI position to the core in the MOJAVE images is so large for these cases, if it locates at different jet components, phase referencing observations can determine the positions of the jet components with sufficient accuracy, which will allow us to locate the VLBI position within the image. This will eventually help us to understand where the Gaia position locates. Figure 7 shows that since the cores of these two sources are not the brightest components, without spectral index images it will be difficult to identify them from radio images, which can lead to a shift of 180 degrees in determining jet position angles.

To conclude, our study suggests that radio source structure is one of the major factors causing the position differences and that the optical jet structure, if it is responsible for the sources with the VLBI-to-Gaia vectors along the radio-jet directions, also tends to be strong for the sources with extended structure at centimeter wavelengths.

\section{Conclusion}

We draw the following conclusion based on the position differences between the Gaia EDR3 and the ICRF3:

1. The arc lengths $\rho$ of the Gaia and VLBI position differences increase with increasing CARMS values.

2. The majority of the sources with statistically significant arc lengths, $X_{\rho}>4$, are associated with extended sources. For instance, the median CARMS of the 432 sources with $X_{\rho}>$ 4 is 0.42 , while that of the remaining 2028 sources is only 0.22 .

3. For the sources with $\rho>4.0$ mas and $X_{\rho}>4$, the majority $(70 \%)$ have extended structure. Source $0429+415$ was used as an example to demonstrate how source structure can cause such large position differences, based on the MOJAVE image shown in Fig. 7.

4. Distinct relations between the optical magnitudes and the redshifts are found for the sources with and without statistically significant position differences. The sources with $X_{\rho}>4$ have substantially smaller redshift values $(\sim 0.3)$. Our study suggests that a small fraction of these sources may be associated with the weak but nearby (at small redshifts) optical objects.

5. We argue that core shift can contribute to the position differences if the source has extended structure.

6. The Gaia and VLBI position differences can be clearly explained through the radio images for several sources as examples. The vectors of the Gaia and VLBI position differences are parallel to the radio-jet directions, which is confirmed with stronger evidence.

In order to construct a more reliable and stable CRF at radio wavelengths, it is necessary and important to mitigate the effects of source structure in astrometric and geodetic VLBI observations. This would then allow us to better detect the true astrophysical differences in the locations of the radio and optical emissions. 
M. H. Xu et al.: Position differences between Gaia and VLBI
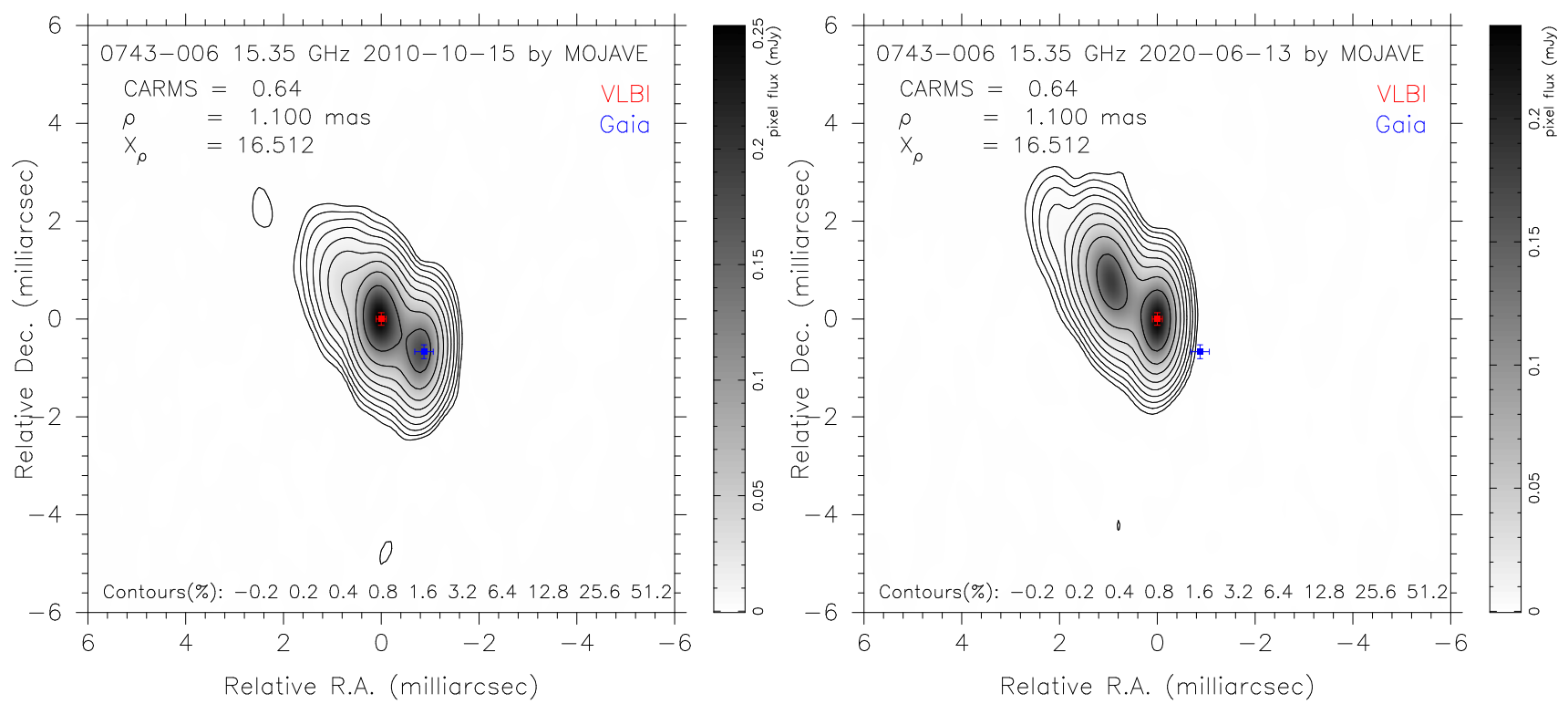

Fig. 6. MOJAVE images of source 0743-006 (CARMS =0.64) at 15 Oct. $2010($ left $)$ and at 13 Jun. 2020 (right). See the caption of Fig. 4 for the plot design. The peak of flux changed between the two components from 2010 to 2020, as indicated by the red dots. Based on its jet motions from model fitting, it was suggested in the MOJAVE project that the source has two-sided jets and the core is located close to the component marked as the red dot in the left plot. It seems possible that the southwest component is the core, meaning that the source has a one-sided jet.
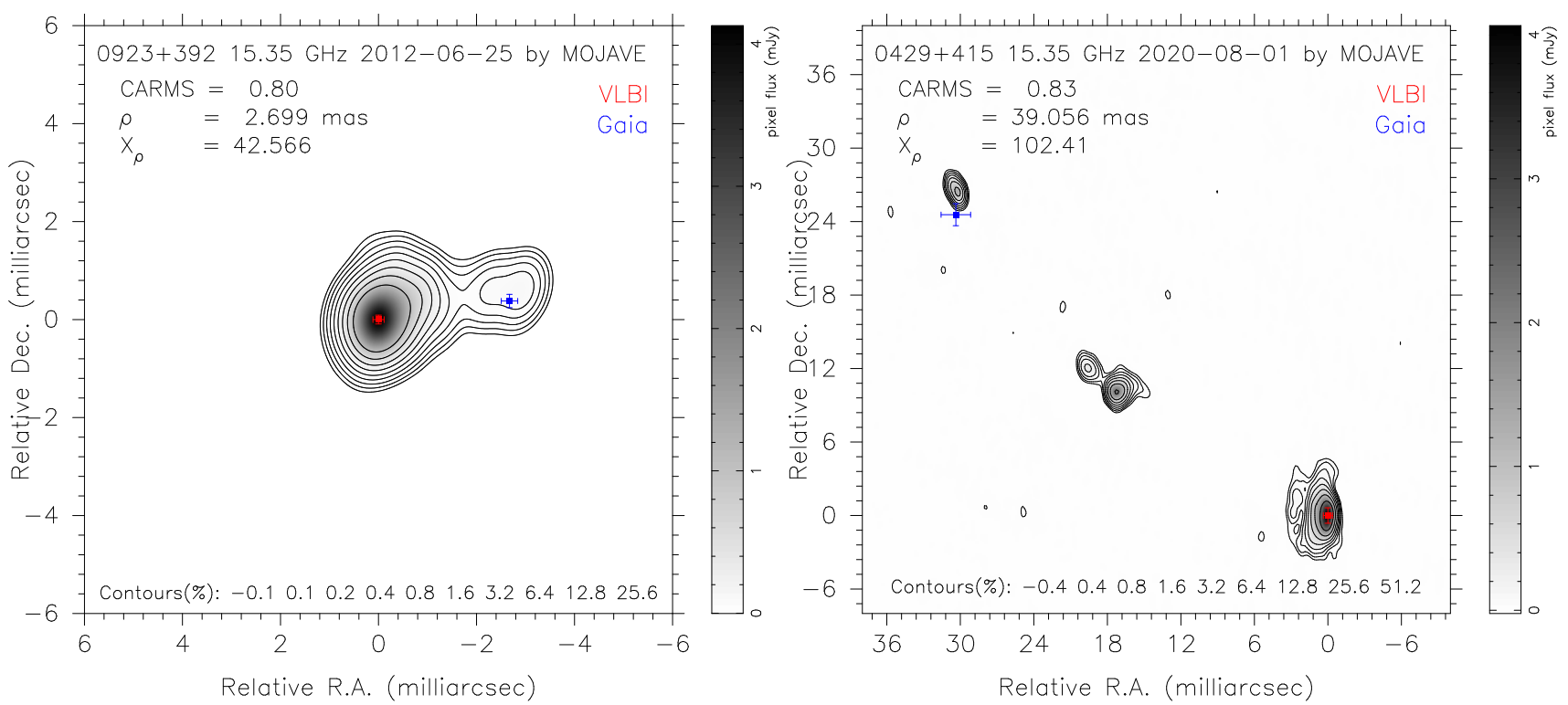

Fig. 7. Explanation of the large Gaia-VLBI position differences for two sources, $0923+392$ with $\rho=2.7$ mas $(4 C 39.25$, CARMS $=0.80$, left $)$ and 0429+415 with $\rho=39.1$ mas (CARMS $=0.83$, right), based on the MOJAVE images (see the caption of Fig. 4 for the plot design). According to the spectral index images from the MOJAVE project, the cores are not the brightest components in the images. The core of the source $0923+392$ is the weak western component, and the core of the source $0429+415$ is the northeastern component. Their Gaia positions are located close to the cores, given that the VLBI positions are located at the peaks of flux. These two sources strongly demonstrate the effects of source structure on the position differences between VLBI and Gaia; the source positions from geodetic VLBI are dominated by the positions of the peak fluxes, whereas the optical positions from Gaia are located close to the cores.

Acknowledgements. We would like to thank the reviewer François Mignard for his helpful comments. This research has made use of data from the MOJAVE database that is maintained by the MOJAVE team (Lister et al. 2018). All components of the International VLBI Service for Geodesy and Astrometry are deeply appreciated for providing the VLBI observations. This research was supported by the Academy of Finland project No. 315721 and the National Natural Science Foundation of China No. 11973023. SL is supported by the DFG grant No. HE593721 We would like to thank the reviewer François Mignard for his helpful comments. This research has made use of data from the MOJAVE database that is maintained by the MOJAVE team (Lister et al. 2018). All components of the International VLBI Service for Geodesy and Astrometry are deeply appreciated for providing the VLBI observations. This research was supported by the Academy of Finland project No. 315721 and the National Natural Science Foundation of China No. 11973023. SL is supported by the DFG grant No. HE59372-2.

\section{References}

Anderson, J. M., \& Xu, M. H. 2018, J. Geophys. Res. (Solid Earth), 123, 162 Arshakian, T. G., Torrealba, J., Chavushyan, V. H., et al. 2010, A\&A, 520, A62 Blandford, R. D., \& Königl, A. 1979, ApJ, 232, 34

Charlot, P. 1990a, A\&A, 229, 51

Charlot, P. 1990b, AJ, 99, 1309 
Charlot, P., Jacobs, C. S., Gordon, D., et al. 2020, A\&A, 644, A159

de Bruijne, J. H. J., Rygl, K. L. J., \& Antoja, T. 2014, in EAS Publications Series, EAS Pub. Ser., 67-68, 23

Fey, A. L., \& Charlot, P. 1997, ApJS, 111, 95

Fey, A. L., Gordon, D., Jacobs, C. S., et al. 2015, AJ, 150, 58

Gaia Collaboration (Brown, A. G. A., et al.) 2016, A\&A, 595, A2

Gaia Collaboration (Brown, A. G. A., et al.) 2018a, A\&A, 616, A

Gaia Collaboration (Mignard, F., et al.) 2018b, A\&A, 616, A14

Gaia Collaboration (Brown, A. G. A., et al.) 2021, A\&A, in press, https://doi.org/10.1051/0004-6361/202039657

Kovalev, Y. Y., Lobanov, A. P., Pushkarev, A. B., \& Zensus, J. A. 2008, A\&A, 483, 759

Kovalev, Y. Y., Petrov, L., \& Plavin, A. V. 2017, A\&A, 598, L1

Kovalev, Y. Y., Zobnina, D. I., Plavin, A. V., \& Blinov, D. 2020, MNRAS, 493, L54

Lindegren, L., Hernández, J., Bombrun, A., et al. 2018, A\&A, 616, A2

Lindegren, L., Klioner, S. A., Hernández, J., et al. 2021, A\&A, in press, https: //doi.org/10.1051/0004-6361/202039709

Lister, M. L., Aller, M. F., Aller, H. D., et al. 2018, ApJS, 234, 12

Lobanov, A. P. 1998, A\&A, 330, 79

Lunz, S., Anderson, J., Heinkelmann, R., Xu, M. H., \& Schuh, H. 2019, in Poster of the 24th European VLBI Group for Geodesy and Astrometry Working Meeting, eds. R. Haas, S. Garcia-Espada, \& J. A. López Fernández

Ma, C., Arias, E. F., Eubanks, T. M., et al. 1998, AJ, 116, 516

Ma, C., Arias, E. F., Bianco, G., et al. 2009, IERS Technical Note, 35, 1

Makarov, V. V., Berghea, C. T., Frouard, J., Fey, A., \& Schmitt, H. R. 2019, ApJ, 873,132
Malkin, Z. 2018, ApJS, 239, 20

Marcaide, J. M., Shapiro, I. I., Corey, B. E., et al. 1985, A\&A, 142, 71

Mignard, F., Klioner, S., Lindegren, L., et al. 2016, A\&A, 595, A5

Niell, A., Whitney, A., Petrachenko, W., et al. 2007, in VLBI2010: a Vision for Future Geodetic VLBI, eds. P. Tregoning, C. Rizos, et al., 757

Nothnagel, A., Artz, T., Behrend, D., \& Malkin, Z. 2017, J. Geod., 91, 711 Perryman, M. A. C., de Boer, K. S., Gilmore, G., et al. 2001, A\&A, 369, 339

Petrachenko, B., Niell, A., Behrend, D., et al. 2009, Design Aspects of the VLBI2010 System. Progress Report of the IVS VLBI2010 Committee, June 2009, Tech. rep.

Petrov, L., \& Kovalev, Y. Y. 2017a, MNRAS, 471, 3775

Petrov, L., \& Kovalev, Y. Y. 2017b, MNRAS, 467, L71

Petrov, L., Kovalev, Y. Y., \& Plavin, A. V. 2019, MNRAS, 482, 3023

Plavin, A. V., Kovalev, Y. Y., \& Petrov, L. Y. 2019a, ApJ, 871, 143

Plavin, A. V., Kovalev, Y. Y., Pushkarev, A. B., \& Lobanov, A. P. 2019b, MNRAS, 485, 1822

Porcas, R. W. 2009, A\&A, 505, L1

Pushkarev, A. B., Hovatta, T., Kovalev, Y. Y., et al. 2012, A\&A, 545, A113

Schuh, H., \& Behrend, D. 2012, J. Geod., 61, 68

Sokolovsky, K. V., Kovalev, Y. Y., Pushkarev, A. B., \& Lobanov, A. P. 2011, A\&A, 532, A38

Xu, M. H., Heinkelmann, R., Anderson, J. M., et al. 2016, AJ, 152, 151

Xu, M. H., Anderson, J. M., Heinkelmann, R., et al. 2019, ApJS, 242, 5

Xu, M. H., Anderson, J. M., Heinkelmann, R., et al. 2021a, J. Geodesy, in press, [arXiv: 2102.12750]

Xu, M. H., Savolainen, T., Zubko, N., et al. 2021b, JGR Solid Earth, in press, https://doi.org/10.1029/2020JB021238 